Supporting Information for

\title{
Polarization of Valence Orbitals by the Intramolecular Electric Field from a Diffuse Dipole-Bound Electron
}

Dao-Fu Yuan, ${ }^{\dagger}$ Yuan Liu, ${ }^{\dagger}$ Chen-Hui Qian, G. Stephen Kocheril, Yue-Rou Zhang, Brenda M. Rubenstein, and Lai-Sheng Wang*

Department of Chemistry, Brown University, Providence, Rhode Island 02912, United States

$\dagger$ ) These authors contributed equally to this work.

*) To whom the correspondence should be addressed: Lai-Sheng_Wang@brown.edu 


\section{Experimental Setup.}

The experiment was carried out using our third-generation electrospray photoelectron spectroscopy apparatus ${ }^{1}$ as shown schematically in Figure S1. The deprotonated 4-(2phenylethynyl)phenoxide anion $\left(\mathrm{PEP}^{-}\right)$was produced by electrospray of a $1 \mathrm{mM}$ solution of 4-(2phenylethynyl)phenol in a mixed solvent of $\mathrm{CH}_{3} \mathrm{OH} / \mathrm{H}_{2} \mathrm{O}(9 / 1$ in volume) at $\mathrm{pH} 10$. Anions generated in the electrospray ionization source were guided into a cryogenically cooled Paul trap operated at $4.6 \mathrm{~K}^{1}$. After being accumulated for $0.1 \mathrm{~s}$ and thermally cooled via collisions with 1 mTorr $\mathrm{He} / \mathrm{H}_{2}$ (4/1 in volume) background gas $^{2}$, the anions were pulsed out at a $10 \mathrm{~Hz}$ repetition rate into the extraction zone of a time-of-flight mass spectrometer. The $\mathrm{PEP}^{-}$anions were selected by a mass gate and photodetached in the interaction zone of a velocity map imaging lens ${ }^{3}$ by the fundamental or the frequency-doubled output of a tunable dye laser. The dye laser was pumped by the $355 \mathrm{~nm}$ output of a $\mathrm{Nd}: \mathrm{YAG}$ laser, and the polarization direction of the detachment laser was parallel to the imaging plane. Photoelectrons were projected onto a pair of 75-mm diameter microchannel plates coupled to a phosphor screen and captured by a charge-coupled-device camera. The photoelectron (PE) images were inverse-Abel transformed and reconstructed using the programs $\mathrm{pBasex}^{4}$ and BASEX ${ }^{5}$. The PE spectra were calibrated with the known spectra of $\mathrm{Au}^{-}$at different photon energies. The kinetic energy (KE) resolution was $3.8 \mathrm{~cm}^{-1}$ for electrons with $55 \mathrm{~cm}^{-1} \mathrm{KE}$ and about $1.5 \%(\triangle \mathrm{KE} / \mathrm{KE})$ for $\mathrm{KE}$ above $1 \mathrm{eV}$ in the current experiment ${ }^{1}$.

\section{Electron Affinity Determination and Resonant Spectra Assignment.}

Figure 1a in the main text shows the non-resonant $\mathrm{PE}$ spectrum of $\mathrm{PEP}^{-}$at a photon energy slightly above the detachment threshold. The near-threshold peak $\left(0_{0}^{0}\right)$ denotes the detachment transition from the ground vibrational level of $\mathrm{PEP}^{-}$to that of PEP. The $0_{0}^{0}$ peak has a full-widthat-half-maximum of $12 \mathrm{~cm}^{-1}$, yielding an accurate electron affinity (EA) of 21,100 $\pm 6 \mathrm{~cm}^{-1}(2.6161$ $\pm 0.0007 \mathrm{eV}$ ) for neutral PEP.

The resonant PE spectra shown in Figure S5 contain the contribution from two detachment channels: the regular non-resonant photodetachment represented by the weak baseline above the detachment threshold in Figure $2 \mathrm{a}$ and the resonantly enhanced autodetachment via single or combinational vibrational levels of the DBS. The vibrational structures in the non-resonant PE spectra are determined by the Franck-Condon (FC) principle, as shown by the FC simulations in 
Figure S3. Autodetachment from DBS to final neutral states follows the $\Delta v=-1$ propensity rule $6,7,8$, i.e., for a single mode excitation, the $n$-th vibrational level of a given mode $v_{x}^{\prime}\left(v_{\mathrm{x}}^{\prime \prime}\right)$ of the DBS autodetaches to the $(n-1)$-th level of the corresponding neutral mode $\left(v_{\mathrm{x}}^{\mathrm{n}-1}\right)$. The corresponding $v_{\mathrm{x}}^{\mathrm{n}-1}$ vibrational peak will be enhanced relative to that in the non-resonant PE spectrum, giving rise to the mode selectivity and highly non-FC PE spectra $^{6,9,10,11,12}$. Similarly, for the excitation to a combinational vibrational level $\left(\mathrm{v}_{\mathrm{x}}^{\prime} \mathrm{v}_{\mathrm{y}}^{\prime n} \ldots\right)$ of the DBS, the final neutral level from autodetachment can be either $v_{\mathrm{x}}^{\mathrm{m}-1} \mathrm{v}_{\mathrm{y}}^{\mathrm{n}} \ldots$ or $\mathrm{v}_{\mathrm{x}}^{\mathrm{m}} \mathrm{v}_{\mathrm{y}}^{\mathrm{n}-1} \ldots$, based on the $\Delta \mathrm{v}=-1$ propensity rule and mode selectivity. This propensity of autodetachment is based on the harmonic oscillator parity selection rule and the fact that the molecular geometry of the DBS is similar to that of the neutral core ${ }^{7,8}$. Due to the mode selectivity and the propensity rule in the autodetachment process, certain vibrational levels are enhanced in the resonant PE spectra, resulting in completely non-FC distributions relative to the non-resonant PE spectra in Figure S3b. All the vibrational levels of the DBS are given in each spectrum in Figure S5, as well as the peak numbers of the corresponding resonant peaks in the photodetachment spectra. The assignments in bold face in Figure S5 indicate the major enhanced vibrational peaks. Most of the assignments of the spectra are fairly straightforward on the basis of the propensity rule. Due to the very different frequencies of the two combinational modes, in the cases of Figure S5a and c-e, only the lower vibrational mode is observed, due to the vibronic coupling of the higher frequency mode with the DBS electron.

\section{Electronic Structure Calculations.}

All the geometry optimizations and ground-state electronic structure calculations are performed using density functional theory (DFT) at the B3LYP/Def2-TZVPP level of theory. The excited states are obtained using time-dependent density function theory (TDDFT) with the same functional and basis set as the ground state calculation. The electronic structure calculations are followed by vibrational, electrostatic, and polarizability analyses. All electronic structure calculations are performed using Gaussian 09 package $^{13}$.

To quantify the influence of the dipole-bound electron to the valence orbitals of the neutral PEP molecule, we position a negative point charge $-e$ along the long axis of the molecule (the same axis as the dipole moment) on the positive side of the dipole moment with varying distance from the center of the molecule (inset 1 in Figure $4 \mathrm{~b}$ of the main text), and then calculate the electronic 
structure of the neutral PEP molecule under the influence of the external electric field produced by this point charge. The resulting valence Kohn-Sham orbitals occupied by a spin-down electron, HOMO-1', HOMO-2', and HOMO-3' are extracted (we use prime to denote the orbitals in the

presence of the external negative point charge) and then project them (inner product) onto the HOMO occupied by the spin-down electron of neutral PEP without the external negative point charge. This procedure allows us to obtain the mixing coefficient $c_{1}$. Moreover, we also project the Kohn-Sham orbitals in the presence of the $-e$ charge onto their corresponding orbitals without the $-e$ charge to obtain the coefficient $c_{0}$. These calculations are all performed with the optimized neutral geometry without the point charge $-e$ at the B3LYP/Def2-TZVPP level of theory. Note that our approach here of representing the DBS electron as a negative point charge is only an approximation. Specifically, the spatial extend as well as the exchange interaction between the DBS electron and the valence electrons are neglected. Nevertheless, this simplified approach captures the essential physics of the valence configuration mixing induced by the oriented intramolecular electric field due to the DBS electron, as shown in Figure 4.

\section{Anisotropy Parameter Calculations.}

The single-photon detachment differential cross section of randomly oriented molecules under linearly-polarized light can be expressed as:

$$
\frac{d \sigma}{d \Omega}=\frac{\sigma_{T}}{4 \pi}\left[1+\beta P_{2}(\cos \theta)\right]
$$

where $\sigma_{T}$ is the total detachment cross section, $-1<\beta<2$ is the anisotropy parameter describing the angular distribution of photoelectrons. $P_{2}$ is the second-order Legendre polynomial and $\theta$ is the angle between the ejected photoelectron and the polarization direction of the detachment laser. $\beta=0$ results in an isotropic $s$-wave detachment, while $\beta=-1$ gives rise to an $(s+d)$-wave scattering and the maximum detachment direction happens perpendicularly to the laser polarization. $\beta=2$ represents a $p$-wave scattering with an angular distribution parallel to the laser polarization.

The $\sigma_{T}$ and $\beta$ parameters can be calculated from first principles using perturbation theory. In the current work, we calculate the anisotropy parameter $\beta$ for all the valence detachment processes using the Kohn-Sham molecular orbitals as the initial bound state wave functions, as described previously ${ }^{14}$. Due to the diffuse nature of the dipole-bound orbital, the radial numerical integration 
for the dipole transition amplitude in real space at large distances suffers from divergent issues. Hence, the calculation of the anisotropy parameter cannot be accomplished in the same way as described above for the valence orbitals. Instead, we estimate the anisotropy parameter $\beta$ for the $X_{\mathrm{DBS}}$ and $B$ DBS transitions (see Figure 2c) using a simplified model based on atomic orbitals as described below. From the Wigner-Beth-Cooper-Zare equation ${ }^{15}$, the anisotropy parameter $\beta$ is given by

$$
\beta=\frac{l(l-1)+(l+1)(l+2) A^{2} \varepsilon^{2}-6 l(l+1) A \varepsilon \cos \left(\delta_{l+1}-\delta_{l-1}\right)}{(2 l+1)\left[l+(l+1) A^{2} \varepsilon^{2}\right]}
$$

where $l$ is the angular momentum of the parent bound state, $A$ is a constant describing the ratio between the radial integrals of the electric-dipole transition amplitudes, i.e., $l \rightarrow l+1$ and $l \rightarrow l-$ 1 , and $\varepsilon$ represents the kinetic energies of the photoelectrons. $\delta_{l+1}-\delta_{l-1}$ is the phase shift difference between the two detachment channels. For a perfect $s$-type orbital $(l=0)$, Eq. (S2) predicts $\beta=2$ which is independent of the kinetic energy $\varepsilon$. When the initial bound state wave function is close to a $s$-type orbital, but with non-negligible $p$ characters, the resulting $\beta$ value monotonically increases with $\varepsilon^{16}$, which agrees with our observation that the $\beta$ value increases from 0.9 for $B_{\mathrm{DBS}}$ to 1.7 for DBS when the electrons kinetic energy is increased by about $2.16 \mathrm{eV}$.

\section{The Transition Probability for the One-photon Two-electron Process.}

Here we describe how the relative intensities of the observed $B_{\mathrm{DBS}}$ and $X_{\mathrm{DBS}}$ peaks in Figure

$2 \mathrm{c}$ are related to the configuration mixing coefficient $c_{0}$ and $c_{1}, I_{B_{D B S}} / I_{X_{D B S}} \approx\left|c_{1}\right|^{2} /\left|c_{0}\right|^{2}$. The electric-dipole transition amplitude $M_{\boldsymbol{k}}$ of detachment from the dipole-bound anion to the neutral PEP final state and a free electron with linear momentum $\boldsymbol{k}$ can be written as:

$M_{k}=C\left\langle\Psi_{v a l}^{k}\left(\boldsymbol{r}_{1}, \boldsymbol{r}_{2}, \ldots, \boldsymbol{r}_{N}\right) \phi_{k}\left(\boldsymbol{r}_{N+1}\right)\left|\varepsilon \cdot\left(\boldsymbol{\mu}_{v a l}+\boldsymbol{\mu}_{D B S}\right)\right| \Psi_{v a l}^{\prime}\left(\boldsymbol{r}_{1}, \boldsymbol{r}_{2}, \ldots, \boldsymbol{r}_{N}\right) \phi_{D B S}\left(\boldsymbol{r}_{N+1}\right)\right\rangle$

where $\boldsymbol{\mu}_{\boldsymbol{v} a l}=e \sum_{i=1}^{N} \boldsymbol{r}_{\boldsymbol{i}}$ and $\boldsymbol{\mu}_{\boldsymbol{D} B S}=e \boldsymbol{r}_{\boldsymbol{N}+1}$ are the dipole operators of the valence electrons and the DBS electron, respectively. $\phi_{D B S}$ is the dipole-bound orbital and $\Psi_{v a l}^{\prime}$ is the wave function of the neutral core in the DBS; $\phi_{\boldsymbol{k}}$ is the wave function of the detached electron in the continuum with momentum $\boldsymbol{k}$ and $\Psi_{v a l}^{\boldsymbol{k}}$ is wave function of the final state. $\boldsymbol{r}_{\mathbf{1}}, \boldsymbol{r}_{2}, \ldots, \boldsymbol{r}_{\boldsymbol{N}}$ stand for the coordinates of the $\mathrm{N}$ valence electrons of the neutral PEP, $\boldsymbol{r}_{N+1}$ represents the coordinates of the dipole-bound electron, and $C$ is a constant. We can further split the contributions from the valence electrons and the DBS electron to rewrite the above equation as 


$$
M_{\boldsymbol{k}}=C\left\langle\Psi_{v a l}^{\boldsymbol{k}}\left|\boldsymbol{\varepsilon} \cdot \boldsymbol{\mu}_{v a l}\right| \Psi_{v a l}^{\prime}\right\rangle\left\langle\phi_{\boldsymbol{k}} \mid \phi_{D B S}\right\rangle+C\left\langle\phi_{\boldsymbol{k}}\left|\boldsymbol{\varepsilon} \cdot \boldsymbol{\mu}_{D B S}\right| \phi_{D B S}\right\rangle\left\langle\Psi_{v a l}^{\boldsymbol{k}} \mid \Psi_{v a l}^{\prime}\right\rangle
$$

where we have omitted the electronic coordinates. In the eq. (S4), the first term represents an electric-dipole transition of the valence electrons of the neutral PEP with an additional factor denoting the overlap between the DBS wave function $\phi_{D B S}$ and the free-electron wave function $\phi_{\boldsymbol{k}}$. The dipole transition element $\left\langle\Psi_{v a l}^{\boldsymbol{k}}\left|\boldsymbol{\varepsilon} \cdot \boldsymbol{\mu}_{\boldsymbol{v a l}}\right| \Psi_{v a l}^{\prime}\right\rangle$ between the two valence states, however, is negligible given the odd parity of the dipole operator $\boldsymbol{\varepsilon} \cdot \boldsymbol{\mu}_{\boldsymbol{v} \boldsymbol{a l}}$ as well as the similarity between $\Psi_{v a l}^{\prime}$ and $\Psi_{v a l}^{k}$. As a result, the first term can be ignored. The second term in eq. (S4) represents the detachment of the DBS electron via an electric-dipole transition into the continuum, multiplied by $\left\langle\Psi_{v a l}^{k} \mid \Psi_{v a l}^{\prime}\right\rangle$ denoting the overlap between the valence electronic states of PEP in the DBS and the final neutral state. The kinetic energy $E_{\boldsymbol{k}}$ of the detached electron is given by its linear momentum $\boldsymbol{k}$ as $E_{\boldsymbol{k}}=\frac{\hbar^{2} k^{2}}{2 m_{e}}$, where $m_{e}$ is the mass of the electron and $\hbar$ is the reduced Planck's constant. Depending on the final state of the neutral core $\Psi_{v a l}^{k}$ (i.e., either in the ground state or an excited states), the momentum (kinetic energy) of the detached electron is different, resulting in distinct peaks (i.e. $X_{\mathrm{DBS}}$ and $B_{\mathrm{DBS}}$ ) in the R2PD spectra in Figure 2. If we denote the electron's momentum as $\boldsymbol{k}_{\mathbf{0}}$ and $\boldsymbol{k}_{\mathbf{1}}$ for the $X_{\mathrm{DBS}}$ and $B \mathrm{DBS}$ peaks, respectively, we can write their transition amplitudes as

$$
\begin{aligned}
& M_{\boldsymbol{k}_{\mathbf{0}}}=C\left\langle\phi_{\boldsymbol{k}_{\mathbf{0}}}\left|\boldsymbol{\varepsilon} \cdot \boldsymbol{\mu}_{D B S}\right| \phi_{D B S}\right\rangle\left\langle\Psi_{v a l}^{\boldsymbol{k}_{\mathbf{0}}} \mid \Psi_{v a l}^{\prime}\right\rangle \\
& M_{\boldsymbol{k}_{\mathbf{1}}}=C\left\langle\phi_{\boldsymbol{k}_{1}}\left|\boldsymbol{\varepsilon} \cdot \boldsymbol{\mu}_{D B S}\right| \phi_{D B S}\right\rangle\left\langle\Psi_{\text {val }}^{\boldsymbol{k}_{\mathbf{1}}} \mid \Psi_{v a l}^{\prime}\right\rangle
\end{aligned}
$$

For the $X_{\mathrm{DBS}}$ peak, the final neutral core is in its ground state, which means $\left|\Psi_{v_{0}}^{\boldsymbol{k}_{\mathbf{0}}}\right\rangle$ can be written as a Slater determinant spanned by a tensor product of the spin-up and spin-down singleelectron wave functions:

$\left|\Psi_{v a l}^{k_{0}}\right\rangle=\left\{\phi_{1 \uparrow}, \phi_{2 \uparrow}, \ldots, \phi_{H O M O-2 \uparrow}, \phi_{H O M O-1 \uparrow}, \phi_{H O M O \uparrow}\right\} \otimes\left\{\phi_{1 \downarrow}, \phi_{2 \downarrow}, \ldots, \phi_{H O M O-2 \downarrow}, \phi_{H O M O-1 \downarrow}\right\}$

Note that the HOMO is only singly occupied by a spin-up electron. Similarly, for the $B$ DBs peak, the spin-down electron on HOMO-2 is excited to the HOMO, leading to a Slater determinant of the form:

$\left|\Psi_{v a l}^{k_{1}}\right\rangle=\left\{\phi_{1 \uparrow}, \phi_{2 \uparrow}, \ldots, \phi_{H O M O-2 \uparrow}, \phi_{H O M O-1 \uparrow}, \phi_{H O M O \uparrow}\right\} \otimes\left\{\phi_{1 \downarrow}, \phi_{2 \downarrow}, \ldots,-, \phi_{H O M O-1 \downarrow}, \phi_{H O M O \downarrow}\right\}$ 
where "-" stands for the removal of the spin-down electron in HOMO-2.

Critically, we have recognized that the DBS electron may influence the neutral valence orbitals. We designate the valence wave function of PEP in the DBS as $\left|\Psi_{v a l}^{\prime}\right\rangle$, which can be constructed from a new set of Kohn-Sham orbitals, $\phi_{i}{ }^{\prime}$ :

$\left|\Psi_{v a l}^{\prime}\right\rangle=$

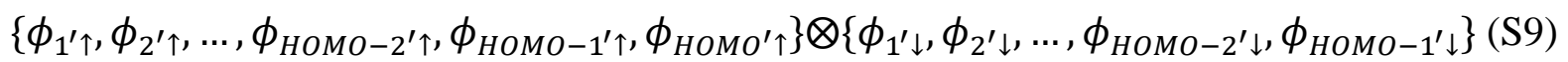

Note that eq. (S9) is similar to eq. (S7) in terms of occupancy, though the shape of each orbital may be changed due to the electric field of the DBS electron. Among all the orbitals in the energy window probed in the current work, the delocalized orbital HOMO-2 is easily deformed to mix with the delocalized HOMO (the same symmetry as HOMO-2). Consequently, HOMO-2' can now be written as a linear combination of HOMO-2 and HOMO:

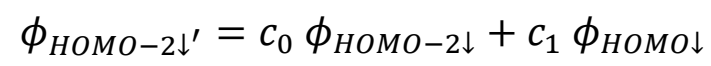

where $c_{0}$ and $c_{1}$ are the mixing coefficients. With these Slater determinants, the overlap between the valence electronic wave functions are evaluated to be simply the overlap between the KohnSham molecular orbitals:

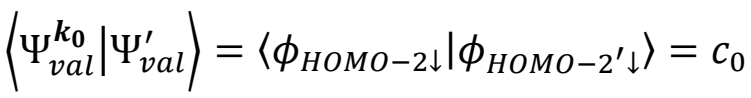

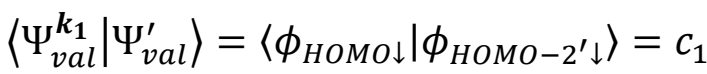

where we have used eq. (S10) to simplify the orbital overlap as mixing coefficients.

Finally, by combining eqs. (S4) and (S5), and eqs. (S11) and (S12), we obtain the ratio between the peak intensity of $B_{\mathrm{DBS}}$ and $X_{\mathrm{DBS}}$ as:

$$
\frac{I_{B_{D B S}}}{I_{X_{D B S}}}=\frac{\left|M_{\boldsymbol{k}_{\mathbf{1}}}\right|^{2}}{\left|M_{\boldsymbol{k}_{\mathbf{0}}}\right|^{2}}=F_{\boldsymbol{k}_{\mathbf{0}}, \boldsymbol{k}_{\mathbf{1}}} \times \frac{\left|c_{1}\right|^{2}}{\left|c_{0}\right|^{2}}
$$

where

$$
F_{\boldsymbol{k}_{0}, \boldsymbol{k}_{1}}=\frac{\left|\left\langle\phi_{\boldsymbol{k}_{1}}\left|\varepsilon \cdot \mu_{D B S}\right| \phi_{D B S}\right\rangle\right|^{2}}{\left|\left\langle\phi_{\boldsymbol{k}_{0}}\left|\varepsilon \cdot \mu_{D B S}\right| \phi_{D B S}\right\rangle\right|^{2}}
$$

The dipole transition term $\left\langle\phi_{\boldsymbol{k}}\left|\boldsymbol{\varepsilon} \cdot \boldsymbol{\mu}_{\boldsymbol{D B S}}\right| \phi_{D B S}\right\rangle$ between the DBS $\phi_{D B S}$ and the free-electron $\phi_{\boldsymbol{k}}$ is generally $\boldsymbol{k}$ dependent. In the current case, both $\boldsymbol{k}_{\mathbf{0}}$ and $\boldsymbol{k}_{\mathbf{1}}$ are large and far from the detachment 
threshold. In the limit of infinite $\boldsymbol{k}_{\mathbf{0}}$ and $\boldsymbol{k}_{\mathbf{1}}$, the difference between the numerator and denominator of eq. (S14) will be negligible, so we can assume $F_{\boldsymbol{k}_{0}, \boldsymbol{k}_{1}}$ to be close to 1 and then eq. (S14) can be further simplified to:

$$
\frac{I_{B_{D B S}}}{I_{X_{D B S}}} \approx \frac{\left|c_{1}\right|^{2}}{\left|c_{0}\right|^{2}}
$$

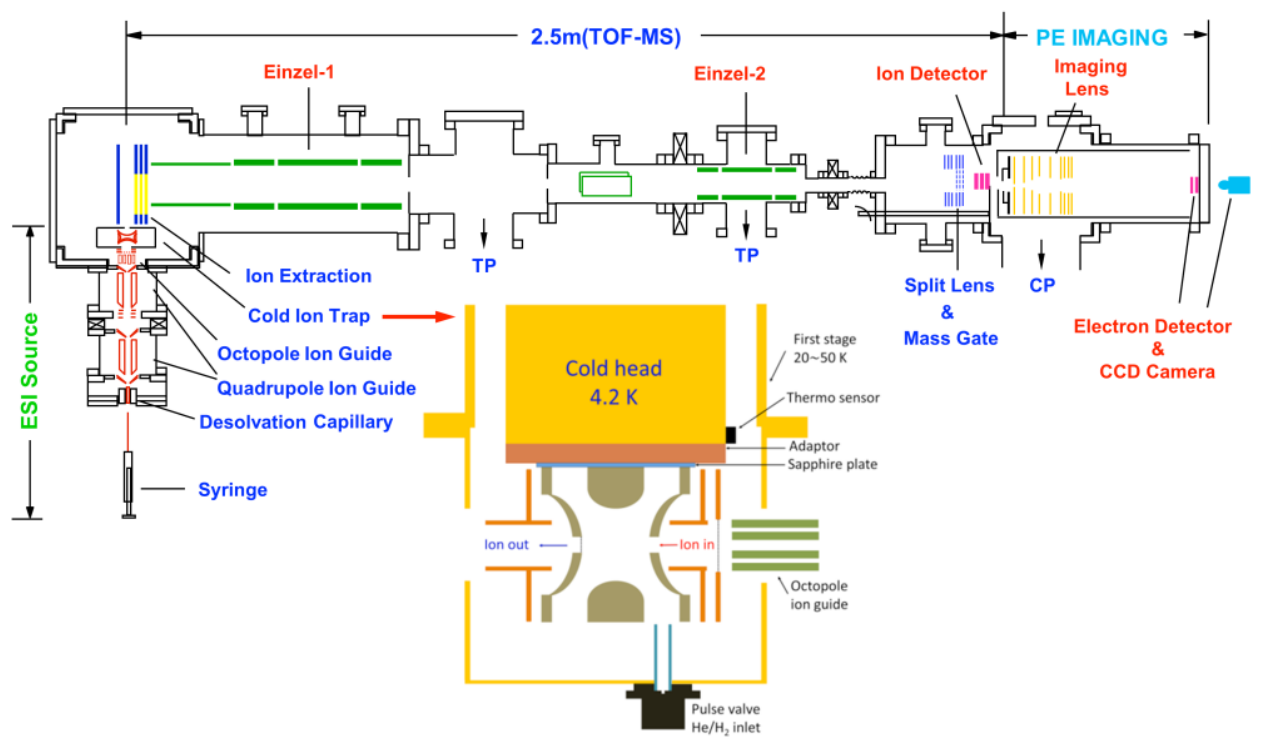

Figure S1. Schematic view of the third-generation high-resolution electrospray photoelectron spectroscopy apparatus ${ }^{17}$. 

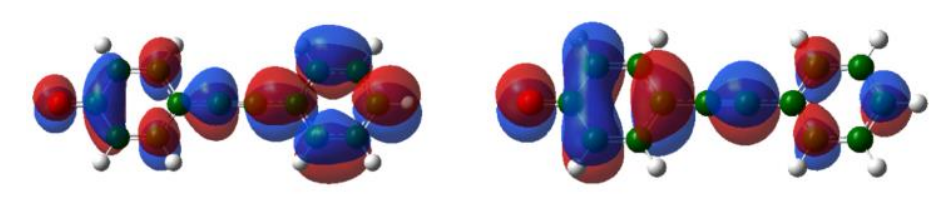

LUMO $\mathbf{b}_{1}$

HOMO $b_{1}$

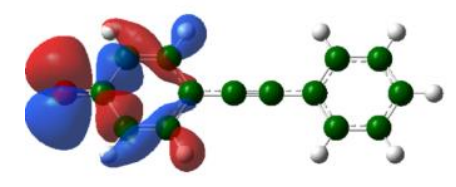

HOMO-1 $b_{2}$

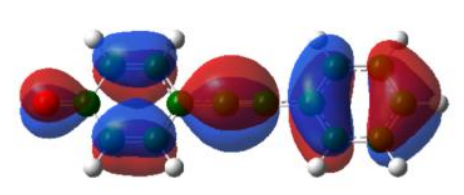

HOMO-2 $b_{1}$

Figure S2. The valence molecular orbitals of the $\mathrm{PEP}^{-}$anion (isovalue $=0.02$ ).

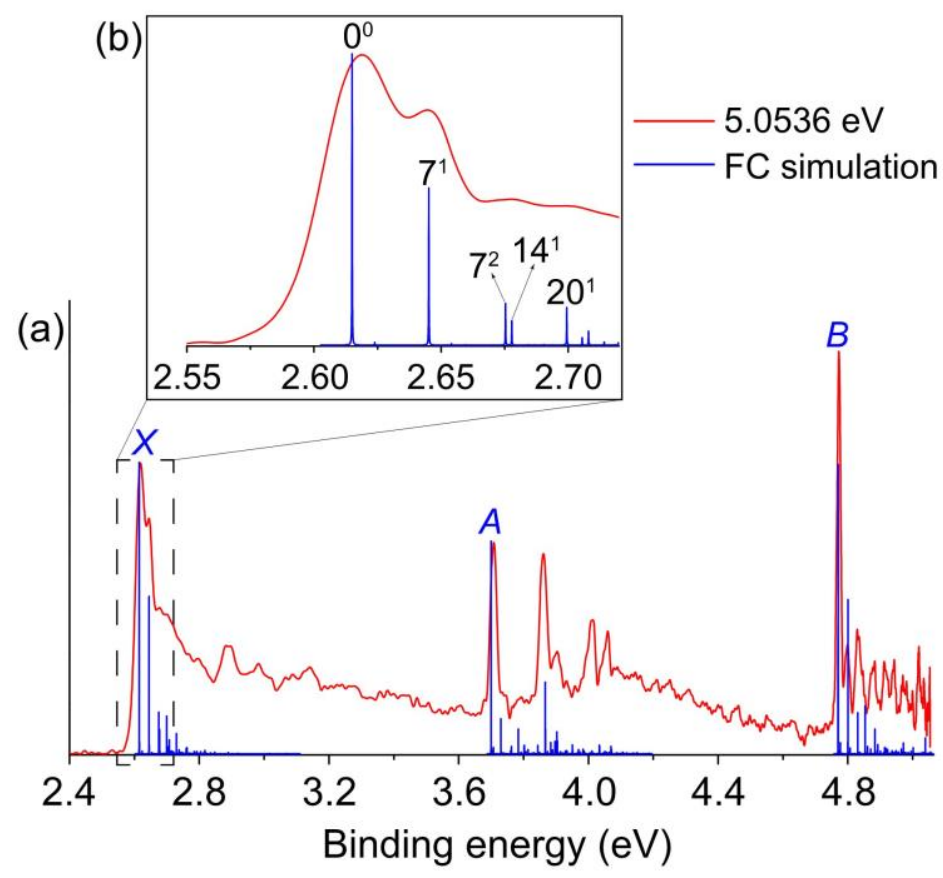

Figure S3. Non-resonant PE spectra of $\mathrm{PEP}^{-}$at $5.0536 \mathrm{eV}$ with the calculated Franck-Condon (FC) factors given as vertical lines for comparison. 
(a)

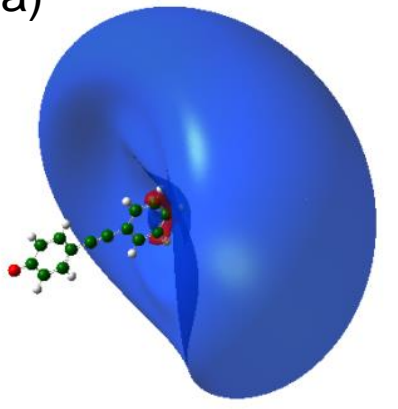

(b)

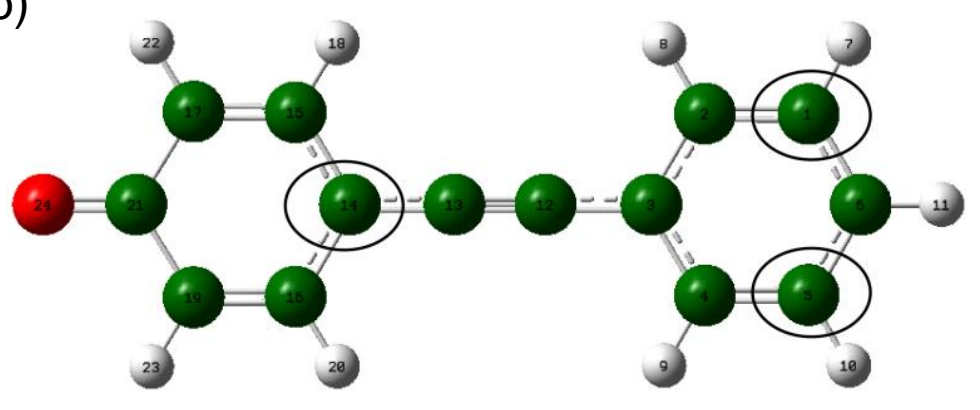

Figure S4. (a) The wave function of the dipole-bound state in the $\mathrm{PEP}^{-}$anion, calculated using the TD-DFT//CAM-B3LYP/Def2-TZVPP+4s3p2d1f level of theory by taking a linear combination of all relevant Kohn-Sham orbitals contributing to the DBS excited state. (b) The additional 4s3p2d1f diffuse basis functions which have been appended to the Def2-TZVPP basis of the three labelled atoms (in black circles) to capture the diffuseness of the DBS. The white, red, and green balls stand for the hydrogen, oxygen and carbon atoms, respectively. The exponents of the diffuse functions are set as 3.942165641E-2, 1.633033298E-2, 6.76480391E-3, 2.80230488E-3 for the 4s functions, 3.501152294E-2, 1.218880611E-2, 4.2433742354E-3 for the 3p functions, 9.21823154E-2 and $2.6721947401 \mathrm{E}-2$ for the $2 \mathrm{~d}$ functions, and $2.536666667 \mathrm{E}-1$ for the $1 \mathrm{f}$ function. The isovalue is chosen to be 0.003 . 

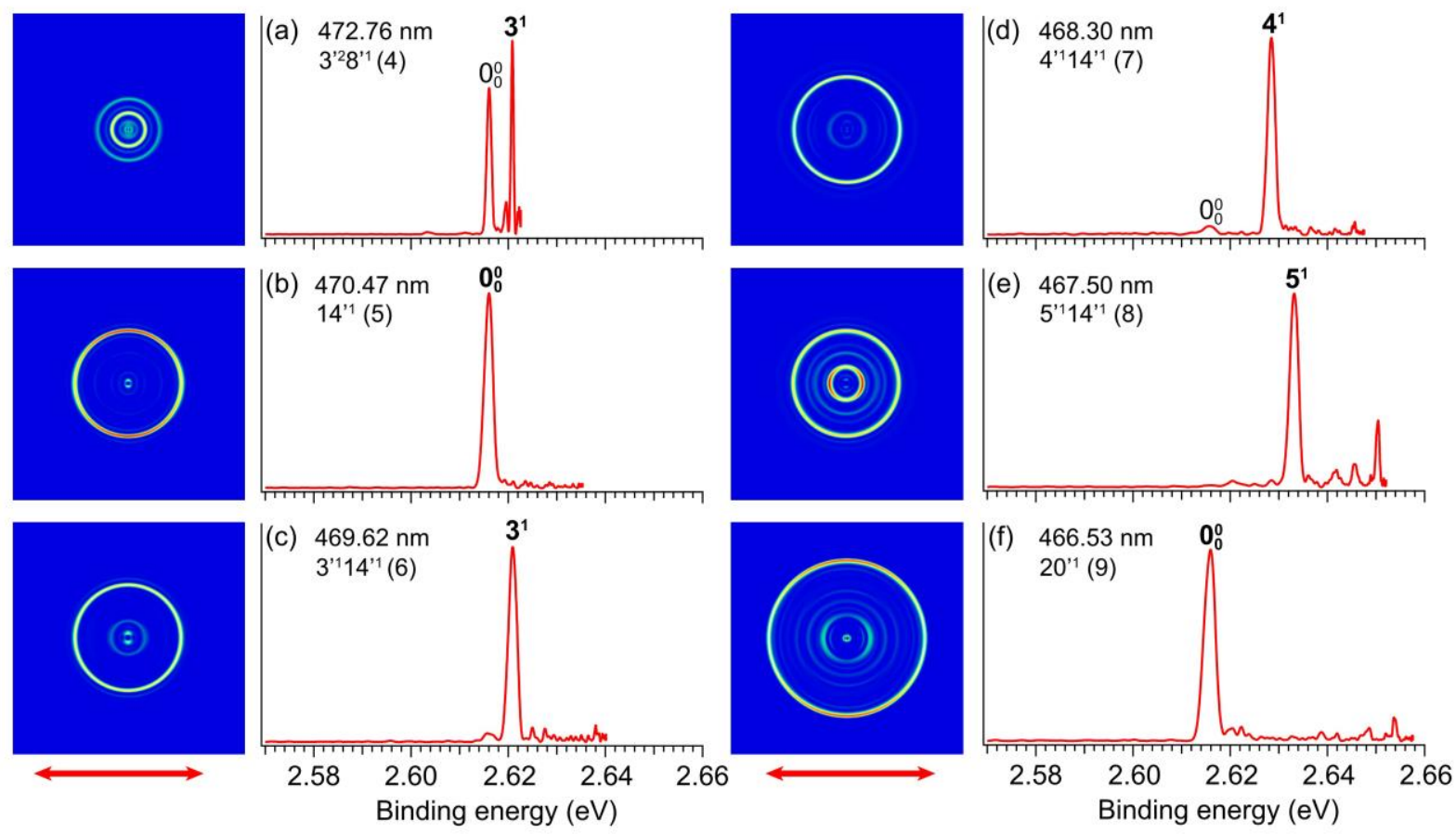

Figure S5. Resonant photoelectron images and spectra at wavelengths corresponding to peaks 49 in Figure 2a (See Table S2 for the photon energies). The assignments of the DBS vibrational levels are shown. The corresponding detachment wavelengths (Table S2) and peak numbers in Figure 2a (in parentheses) are also given. The double arrows below the images indicate the laser polarization. 


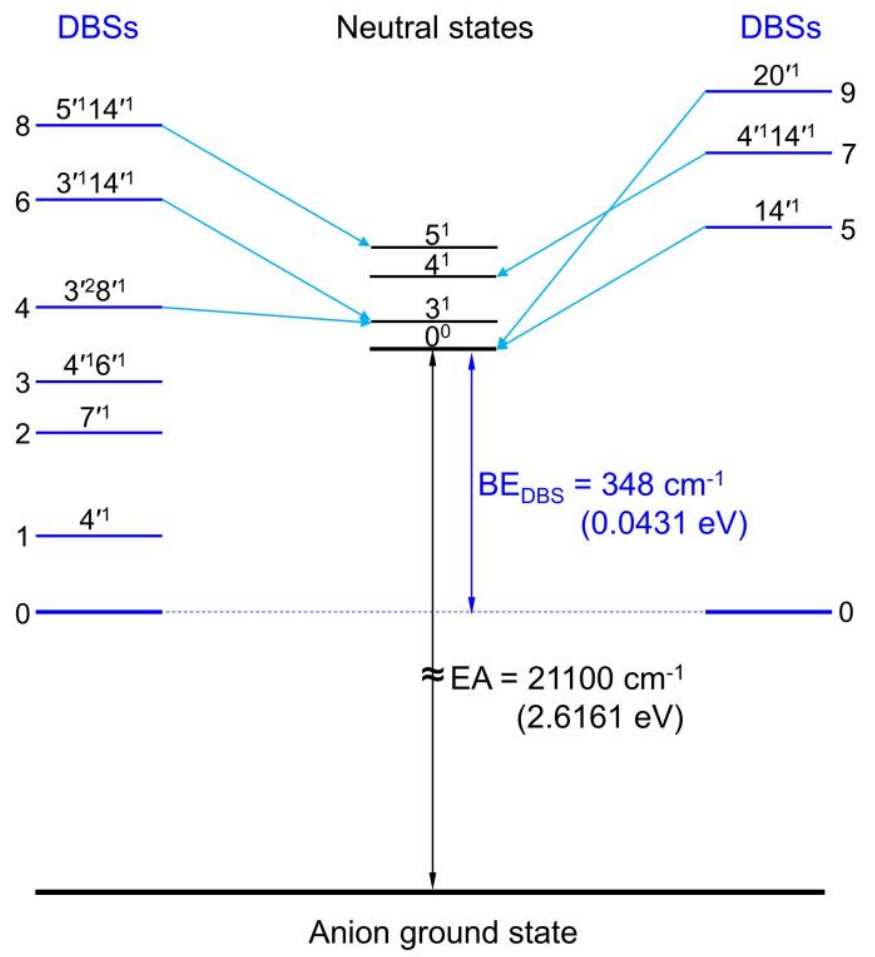

Figure S6. Schematic energy level diagram for autodetachment from the DBS vibrational levels of $\mathrm{PEP}^{-}$to the related neutral final states, corresponding to the ten resonant PE spectra shown in Figure 2c and Figure S5.
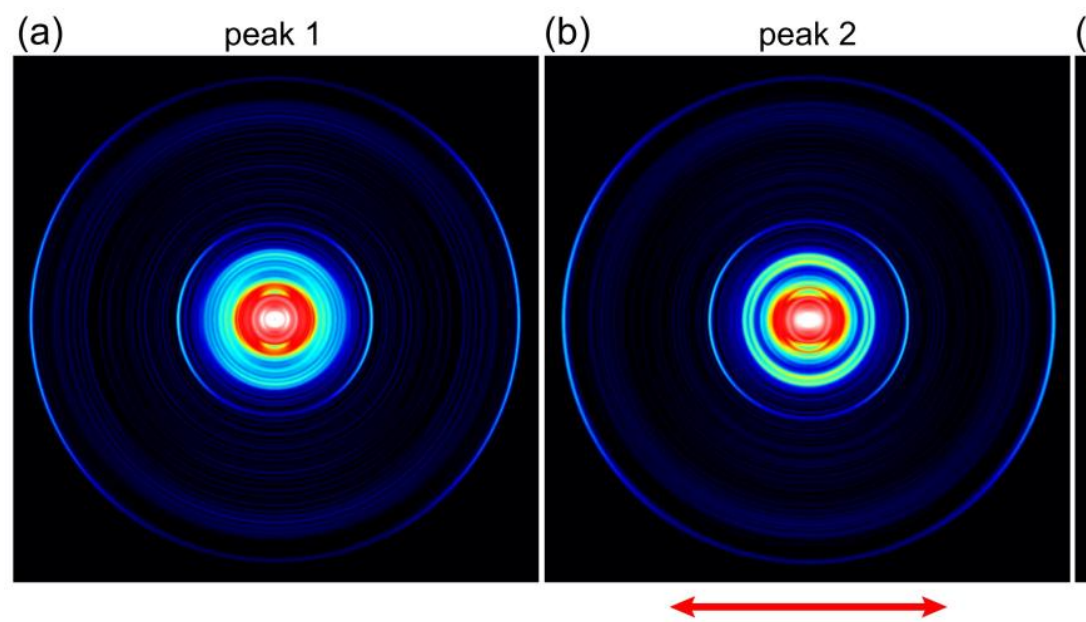

(c) peak 3

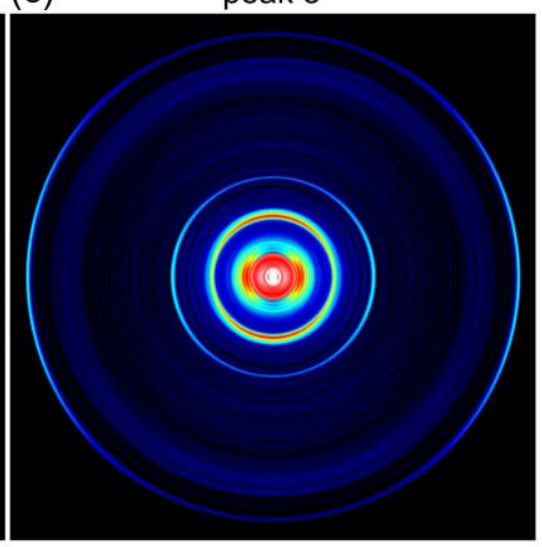

Figure S7. The R2PD PE images of the $\mathrm{PEP}^{-}$anion via the bound vibrational levels of the DBS at (a) 20,854 $\mathrm{cm}^{-1}$ (corresponding to peak 1 in Figure 2a), (b) 20,991 $\mathrm{cm}^{-1}$ (peak 2 in Figure 2a), and (c) $21,057 \mathrm{~cm}^{-1}$ (peak 3 in Figure 2a). See Table S2 for the wavelengths. The double arrow below the image indicates the laser polarization. 


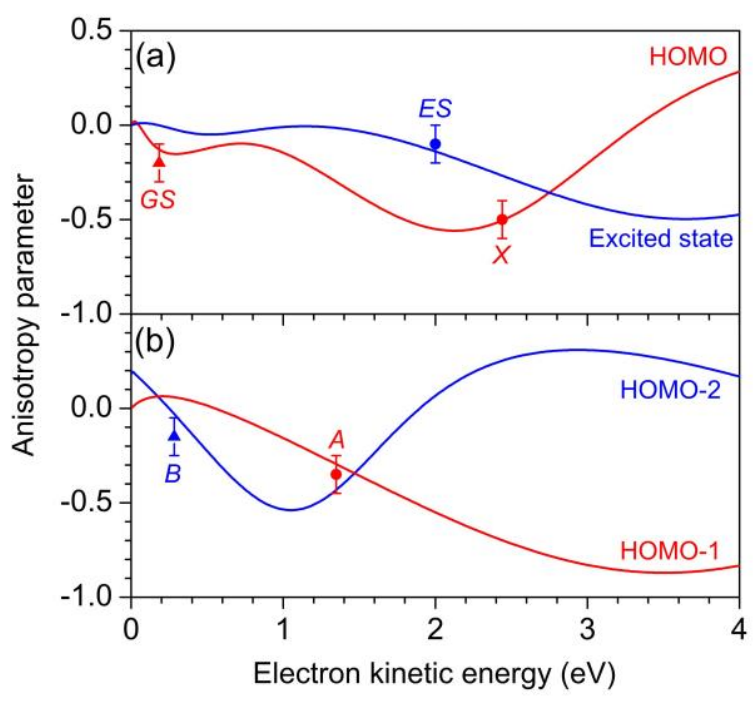

Figure S8. The experimental (symbols) and calculated (solid lines) anisotropy parameters for the photoelectrons detached from (a) HOMO of the $\mathrm{PEP}^{-}$anion's ground (red) and excited state (blue), (b) HOMO-1 (red) and HOMO-2 (blue) of the $\mathrm{PEP}^{-}$anion's ground state. The experimental data points (with error bars) labeled as $E S, G S, X, A$, and $B$ represent the anisotropy parameters of the corresponding peaks labeled in Figures $1(X, A, B)$ and Figure $2(E S, G S)$.

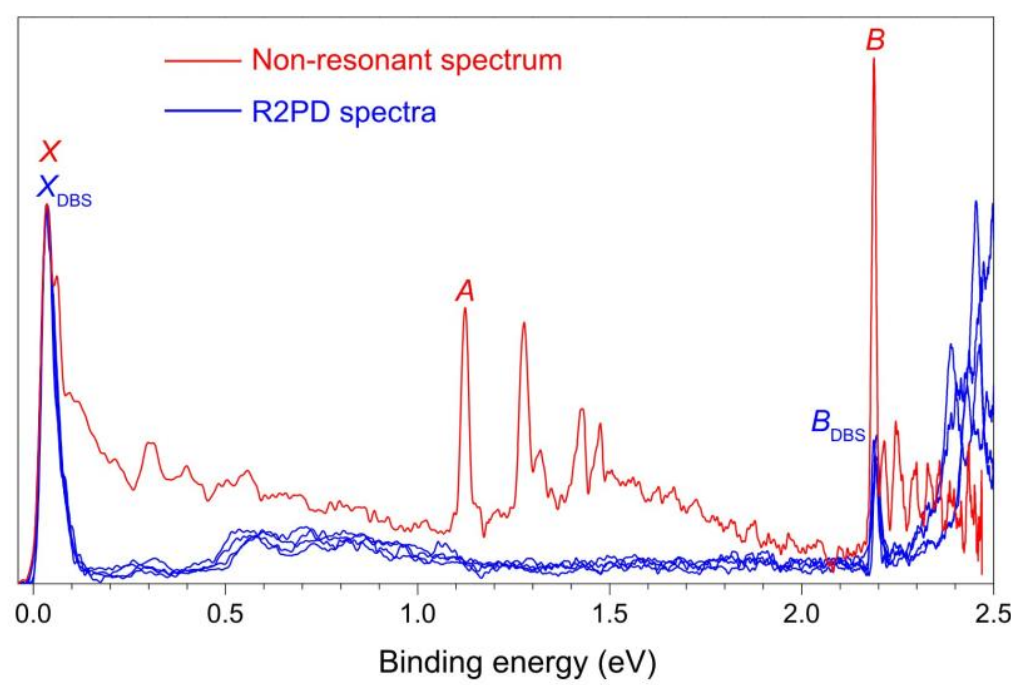

Figure S9. Comparison of the R2PD PE spectra at wave lengths corresponding to peaks 0-3 in Figure 2c (blue traces) and the single-photon non-resonant spectrum in Figure 1d (red trace, shifted to align peaks $X$ and the R2PD $X$ DBS peak). Note the identical separations between peaks $B$ and $X$ and between peaks $B_{\mathrm{DBS}}$ and $X_{\mathrm{DBS}}$. 
(a)

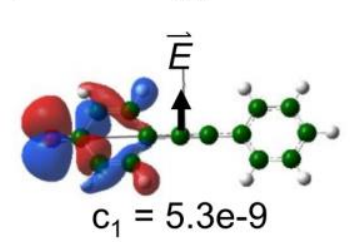

(b)

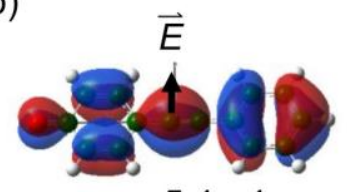

$c_{1}=5.4 \mathrm{e}-4$

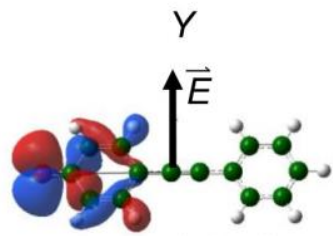

$c_{1}=-6.6 e-9$

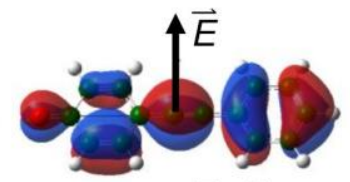

$c_{1}=-7 e-3$
$-Z$
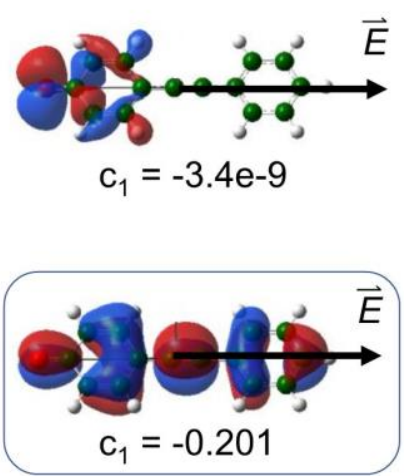

Figure S10. Schematic pictures showing how an electric field along the $X, Y$, and $-Z$ direction polarizes (a) the HOMO-1 and (b) HOMO-2 of PEP. The field is applied with equal magnitude in the three directions in the molecular frame. The configuration mixing coefficient $c_{l}$ is given in each case. The PEP molecule lies within the $Y Z$ plane with the $X$ axis perpendicular to the molecular plane. Note that the applied field has very little effect to the HOMO-1, whereas the field applied in the $-Z$ direction has a large effect to the HOMO-2.

Table S1. The observed adiabatic detachment energies (ADE) of the photoelectron features of the $\mathrm{PEP}^{-}$anion and the excitation energies $(\triangle \mathrm{E})$ of the excited states of neutral PEP, compared with the calculated excitation energies.

\begin{tabular}{cccc}
\hline \hline Observed feature & $\operatorname{ADE}(\mathrm{eV})$ & $\begin{array}{c}\Delta \mathrm{E}(\mathrm{eV}) \\
(\mathrm{exp})\end{array}$ & $\begin{array}{c}\Delta \mathrm{E}(\mathrm{eV}) \\
(\text { theo })^{\mathrm{a}}\end{array}$ \\
\hline$X$ & $2.6161 \pm 0.0007$ & & \\
$A$ & $3.7079 \pm 0.0131$ & 1.092 & 1.14 \\
$B$ & $4.7720 \pm 0.0077$ & 2.156 & 2.21 \\
\hline \hline
\end{tabular}

${ }^{\mathrm{a}}$ Calculated from the time-dependent density functional theory. 
Table S2. Assignments of the observed peaks in the photodetachment spectra of $\mathrm{PEP}^{-}$, along with their wavelengths, photon energies and relative energy shifts to the ground vibrational level of the DBS.

\begin{tabular}{ccccc}
\hline \hline Peak & $\begin{array}{c}\text { Wavelength } \\
(\mathrm{nm})\end{array}$ & $\begin{array}{c}\text { Photon Energy } \\
\left(\mathrm{cm}^{-1}\right)^{\mathrm{a}}\end{array}$ & $\begin{array}{c}\text { Shift } \\
\left(\mathrm{cm}^{-1}\right)\end{array}$ & Assignment \\
\hline 0 & 481.88 & $20752(5)$ & 0 & $\begin{array}{c}\text { vibrational ground } \\
\text { state of the DBS }\end{array}$ \\
1 & 479.52 & $20854(5)$ & 102 & $4^{\prime 1}$ \\
2 & 476.40 & $20991(5)$ & 239 & $7^{\prime 1}$ \\
3 & 474.90 & $21057(5)$ & 305 & $4^{\prime 1} 6^{\prime 1}$ \\
4 & 472.76 & $21152(5)$ & 400 & $3^{\prime 2} 8^{\prime 1}$ \\
5 & 470.47 & $21255(5)$ & 503 & $14^{\prime 1}$ \\
6 & 469.62 & $21294(5)$ & 542 & $3^{\prime 1} 14^{\prime 1}$ \\
7 & 468.30 & $21354(5)$ & 602 & $4^{\prime 1} 14^{\prime 1}$ \\
8 & 467.50 & $21390(5)$ & 638 & $5^{\prime 1} 14^{\prime 1}$ \\
\hline & 466.53 & $21435(5)$ & 682 & $20^{\prime 1}$ \\
\hline
\end{tabular}

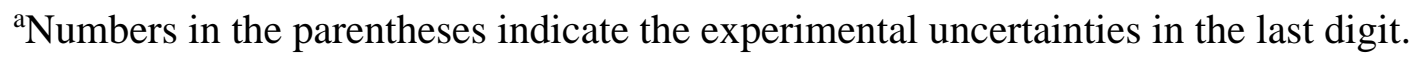


Table S3. Calculated vibrational frequencies for neutral PEP at DFT/ B3LYP/Def2TZVPP level of theory, compared with observed frequencies.

\begin{tabular}{|c|c|c|c|}
\hline $\begin{array}{c}\text { Vibrational } \\
\text { Mode }\end{array}$ & Symmetry & $\begin{array}{c}\text { Theoretical } \\
\text { Frequency }\left(\mathrm{cm}^{-1}\right)\end{array}$ & $\begin{array}{c}\text { Experimental } \\
\text { Frequency }\left(\mathrm{cm}^{-1}\right)\end{array}$ \\
\hline$v_{1}$ & $A_{2}$ & 36 & 30 \\
\hline$v_{2}$ & $B_{1}$ & 41 & 36 \\
\hline$v_{3}$ & $B_{2}$ & 43 & 39 \\
\hline$v_{4}$ & $B_{1}$ & 105 & 102 \\
\hline$v_{5}$ & $B_{2}$ & 148 & 135 \\
\hline$v_{6}$ & $B_{1}$ & 201 & 203 \\
\hline$v_{7}$ & $A_{1}$ & 243 & 239 \\
\hline$v_{8}$ & $B_{1}$ & 318 & 322 \\
\hline$v_{9}$ & $A_{2}$ & 386 & \\
\hline$v_{10}$ & $A_{2}$ & 412 & \\
\hline$v_{11}$ & $B_{2}$ & 431 & \\
\hline$v_{12}$ & $B_{1}$ & 435 & \\
\hline$v_{13}$ & $B_{2}$ & 490 & \\
\hline$v_{14}$ & $A_{l}$ & 507 & 503 \\
\hline$v_{15}$ & $B_{1}$ & 528 & \\
\hline$v_{16}$ & $B_{2}$ & 567 & \\
\hline$v_{17}$ & $B_{1}$ & 567 & \\
\hline$v_{18}$ & $B_{2}$ & 633 & \\
\hline$v_{19}$ & $B_{2}$ & 642 & \\
\hline$v_{20}$ & $A_{1}$ & 681 & 682 \\
\hline$v_{21}$ & $B_{1}$ & 705 & \\
\hline$v_{22}$ & $B_{1}$ & 759 & \\
\hline$v_{23}$ & $B_{1}$ & 782 & \\
\hline$v_{24}$ & $A_{2}$ & 800 & \\
\hline$v_{25}$ & $A_{1}$ & 800 & \\
\hline
\end{tabular}




\begin{tabular}{|c|c|c|}
\hline$v_{26}$ & $A_{1}$ & 845 \\
\hline$V_{27}$ & $A_{2}$ & 859 \\
\hline$v_{28}$ & $B_{1}$ & 879 \\
\hline$v_{29}$ & $B_{1}$ & 946 \\
\hline$v_{30}$ & $B_{1}$ & 986 \\
\hline$v_{31}$ & $A_{l}$ & 995 \\
\hline$v_{32}$ & $A_{2}$ & 999 \\
\hline$v_{33}$ & $B_{1}$ & 1000 \\
\hline$v_{34}$ & $B_{1}$ & 1014 \\
\hline$v_{35}$ & $A_{1}$ & 1016 \\
\hline$v_{36}$ & $A_{1}$ & 1049 \\
\hline$v_{37}$ & $B_{2}$ & 1105 \\
\hline$v_{38}$ & $B_{2}$ & 1112 \\
\hline$v_{39}$ & $A_{1}$ & 1139 \\
\hline$V_{40}$ & $A_{1}$ & 1184 \\
\hline$V_{41}$ & $B_{2}$ & 1186 \\
\hline$V_{42}$ & $A_{1}$ & 1206 \\
\hline$V_{43}$ & $B_{2}$ & 1275 \\
\hline$V_{44}$ & $B_{2}$ & 1293 \\
\hline$V_{45}$ & $B_{2}$ & 1313 \\
\hline$V_{46}$ & $B_{2}$ & 1359 \\
\hline$V_{47}$ & $A_{1}$ & 1360 \\
\hline$V_{48}$ & $A_{1}$ & 1446 \\
\hline$V_{49}$ & $B_{2}$ & 1454 \\
\hline$v_{50}$ & $B_{2}$ & 1478 \\
\hline$v_{51}$ & $B_{2}$ & 1502 \\
\hline$v_{52}$ & $A_{1}$ & 1528 \\
\hline$V_{53}$ & $A_{1}$ & 1538 \\
\hline$v_{54}$ & $A_{1}$ & 1601 \\
\hline
\end{tabular}




\begin{tabular}{lll}
$v_{55}$ & $B_{2}$ & 1604 \\
$v_{56}$ & $A_{1}$ & 1635 \\
$v_{57}$ & $A_{1}$ & 2211 \\
$v_{58}$ & $A_{1}$ & 3173 \\
$v_{59}$ & $A_{1}$ & 3181 \\
$v_{60}$ & $B_{2}$ & 3181 \\
$v_{61}$ & $B_{2}$ & 3182 \\
$v_{62}$ & $A_{1}$ & 3192 \\
$v_{63}$ & $B_{2}$ & 3199 \\
$v_{64}$ & $B_{2}$ & 3199 \\
$v_{65}$ & $A_{1}$ & 3200 \\
$v_{66}$ & $A_{l}$ & 3203 \\
\hline \hline
\end{tabular}

Table S4. Polarizability tensor $\alpha$ (atomic units) of the neutral PEP molecule. The off diagonal components are all zero (not displayed here). The results are obtained at the B3LYP/Def2TZVPP level of theory under the optimized neutral geometry.

\begin{tabular}{cc}
\hline \hline Polarizability & PEP \\
\hline$\alpha_{x x}$ & 81.5 \\
$\alpha_{y y}$ & 151.8 \\
$\alpha_{z z}$ & 432.0 \\
\hline$\alpha_{i s o}$ & 221.76 \\
\hline \hline
\end{tabular}




\section{References}

(1) Wang, L. S. Electrospray Photoelectron Spectroscopy: from Multiply-Charged Anions to Ultracold Anions. J. Chem. Phys. 2015, 143, 040901.

(2) Wang, X. B.; Wang, L. S. Development of a Low-Temperature Photoelectron Spectroscopy Instrument Using an Electrospray Ion Source and a Cryogenically Controlled Ion Trap. Rev. Sci. Instrum. 2008, 79, 073108.

(3) León, I.; Yang, Z.; Liu, H. T.; Wang, L. S. The Design and Construction of a High-Resolution Velocity-Map Imaging Apparatus for Photoelectron Spectroscopy Studies of Size-Selected Clusters. Rev. Sci. Instrum. 2014, 85, 083106.

(4) Garcia, G. A.; Nahon, L.; Powis, I. Two-Dimensional Charged Particle Image Inversion Using a Polar Basis Function Expansion. Rev. Sci. Instrum. 2004, 75, 4989-4996.

(5) Dribinski, V.; Ossadtchi, A.; Mandelshtam, V. A.; Reisler, H. Reconstruction of AbelTransformable Images: the Gaussian Basis-Set Expansion Abel Transform Method. Rev. Sci. Instrum. 2002, 73, 2634-2642.

(6) Zhu, G. Z.; Wang, L. S. High-Resolution Photoelectron Imaging and Resonant Photoelectron Spectroscopy via Noncovalent-Bound Excited States of Cryogenically-Cooled Anions. Chem. Sci. 2019, 10, 9409-9423.

(7) Berry, R. S. Ionization of Molecules at Low Energies. J. Chem. Phys. 1966, 45, 1228-1245.

(8) Simons, J. Propensity Rules for Vibrational-Induced Electron Detachment of Anions. J. Am. Chem. Soc. 1981, 103, 3971-3976.

(9) Liu, H. T.; Ning, C. G.; Huang, D. L.; Dau, P. D.; Wang, L. S. Observation of Mode-Specific Vibrational Autodetachment from Dipole-Bound States of Cold Anions. Angew. Chem. Int. Ed. 2013, 52, 8976-8979.

(10) Liu, H. T.; Ning, C. G.; Huang, D. L.; Wang, L. S. Vibrational Spectroscopy of the Dehydrogenated Uracil Radical via Autodetachment of Dipole-Bound Excited States of Cold Anions. Angew. Chem. Int. Ed. 2014, 53, 2464-2468.

(11) Huang, D. L.; Ning, C. G.; Liu, H. T.; Wang, L. S. Conformation-Selective Resonant Photoelectron Spectroscopy via Dipole-Bound States of Cold Anions. J. Phys. Chem. Lett. 2015, 6, 2153-2157.

(12) Zhu, G. Z.; Qian, C. H.; Wang, L. S. Tautomer-Specific Resonant Photoelectron Imaging of Deprotonated Cytosine Anions. Angew. Chem. Int. Ed. 2019, 58, 7856-7860. 
(13) Gaussian 09, Revision D.01, Frisch, M. J., Trucks, G. W., Schlegel, H. B., Scuseria, G. E., Robb, M. A., Cheeseman, J. R., Scalmani, G., Barone, V., Petersson, G. A., Nakatsuji, H., Li, X. Caricato, M., Marenich, A., Bloino, J., Janesko, B. G., Gomperts, R., Mennucci, B., Hratchian, H. P., Ortiz, J. V., Izmaylov, A. F., Sonnenberg, J. L., Williams-Young, D., Ding, F., Lipparini, F., Egidi, F., Goings, J., Peng, B., Petrone, A., Henderson, T., Ranasinghe, D., Zakrzewski, V. G., Gao, J., Rega, N., Zheng, G., Liang, W., Hada, M., Ehara, M., Toyota, K., Fukuda, R., Hasegawa, J., Ishida, M., Nakajima, T., Honda, Y., Kitao, O., Nakai, H., Vreven, T., Throssell, K., Montgomery, J. A. Jr., Peralta, J. E., Ogliaro, F., Bearpark, M., Heyd, J. J., Brothers, E., Kudin, K. N., Staroverov, V. N., Keith, T., Kobayashi, R., Normand, J., Raghavachari, K., Rendell, A., Burant, J. C., Iyengar, S. S., Tomasi, J., Cossi, M., Millam, J. M., Klene, M., Adamo, C., Cammi, R., Ochterski, J. W., Martin, R. L., Morokuma, K., Farkas, O., Foresman, J. B.; Fox, D. J. Gaussian, Inc., Wallingford CT, 2016.

(14) Liu, Y.; Ning, C. G. Calculation of Photodetachment Cross Sections and Photoelectron Angular Distributions of Negative Ions using Density Functional Theory. J. Chem. Phys. 2015, $143,144310$.

(15) Hanstorp, D.; Bengtsson, C.; Larson, D. J. Angular Distributions in Photodetachment from $\mathrm{O}^{-}$. Phys. Rev. A 1989, 40, 670-675.

(16) Grumbling, E. R.; Sanov, A. Photoelectron Angular Distribution in Negative-Ion Photodetachment from Mixed sp States. J. Chem. Phys. 2011, 135, 164302.

(17) Wang, L. S. Electrospray Photoelectron Spectroscopy: from Multiply-Charged Anions to Ultracold Anions. J. Chem. Phys. 2015, 143, 040901. 